\title{
A MODIFIED PROJECTION METHOD \\ FOR EQUATIONS OF THE SECOND KIND
}

\author{
M. Thamban Nair
}

A modified projection method is suggested for the approximate solution of second kind equations and it is compared with other methods.

\section{Introduction}

Let $T$ be a bounded operator on a Banach space $X$ such that $I$ is not in the spectrum of $T$, and $\left(\pi_{n}\right)$ a sequence of finite rank projections on $X$ such that $\lim _{n \rightarrow \infty} \pi_{n} x=x$ for all $x \in X$. The purpose of this note is to introduce a new approximation method, we call it a modified projection method, to obtain an approximation for the solution of the equation

$$
x-T x=y,
$$

with the help of the projection $\pi_{n}$. It is to be noted that our assumption that 1 does not belong to the spectrum of $T$ implies the existence of a unique solution for (1.1). We discuss the advantage of our method over the well-known approximation methods, namely, the Galerkin method, the iterated Galerkin method of Sloan and the Kantorovich method considered by schock.

20 January 1987. I would like to thank Professor B.V. Limaye for his suggestions about the section entitled "Computational Remarks".

Copyright Clearance Centre, Inc. Serial-fee code: 0004-9729/87 $\$ A 2.00+0.00$. 
The Galerkin approximation $x_{n}^{G}$ is the solution of

$$
x_{n}^{G}-\pi_{n} T x_{n}^{G}=\pi_{n^{y}} \text {. }
$$

The iterated Galerkin approximation (or sloan approximation) $x_{n}^{S}$ is defined by

$$
x_{n}^{S}=y+T x_{n}^{G} \text {. }
$$

The Kantorovich method considered by Schock $[4,5,6]$ determines an approximation $x_{n}^{K}$ as

$$
x_{n}^{K}=y+z_{n},
$$

where $z_{n}$ is the solution of

$$
z_{n}-\pi_{n} T z_{n}=\pi_{n} T y \text {. }
$$

It can be verified that $x_{n}^{S}$ and $x_{n}^{K}$ satisfy the following equations:

$$
\begin{aligned}
& x_{n}^{S}-T \pi_{n} x_{n}^{S}=y \\
& x_{n}^{K}-\pi_{n} T x_{n}^{K}=y .
\end{aligned}
$$

It is known that under certain conditions (for example, if $T$ is a compact operator on a Hilbert space $X$ and $\pi_{n}$, is an orthogonal projection), the iterated Galerkin approximation $x_{n}^{S}$ converges to the solution $x$ of (1.1) faster than $x_{n}^{G}$ (see sloan [7] and also sloan, Noussair and Burn [8]). Also, if $T$ is an integral operator

$$
(T x)(s)=\int_{a}^{b} k(s, t) x(t) d t, a \leq s \leq b
$$

on a suitable function space, then, under some reqularity conditions on $y$ and the kernel $k, x_{n}^{S}$ has better convergence rates than $x_{n}^{G}$ (see Chandler [1]). It is observed in Schock [5] (see also [4]) that if $T$ is a 'smoothing' operator then the Kantorovich approximation $x_{n}^{K}$ is superior to both the Galerkin approximation $x_{n}^{G}$ and the iterated Galerkin approximation $x_{n}^{S}$. Also, in a recent paper, schock [6] showed that the Kantorovich method has a better global convergence behaviour than the 
Galerkin and iterated Galerkin methods. But the disadvantage of the iterated Galerkin and the Kantorovich methods is that in one case we have to calculate $T x_{n}{ }^{G}$ and in the other $\pi n^{T y}$ - both these, in practice, would introduce some extra errors in the approximation when they are calculated by numerical methods. To overcome this difficulty we define a new (modified projection) approximation $x_{n}^{M}$ as

$$
x_{n}^{M}=x_{n}^{G}+\left(1-\pi_{n}\right) y \text {. }
$$

It should be noted that for the computation of $x_{n}{ }^{G}$ it is necessary to know $\pi n^{y}$ (see (1.2)). Thus $x_{n}^{M}$ involves no more computation than $x_{n}^{G}$. The definition of $x_{n}^{M}$ is motivated by the expression for $x_{n}{ }^{S}$ in (1.3): $x_{n}^{M}$ is obtained from (1.3) by replacing $T x_{n}^{G}$ by $\pi_{n} T x_{n}^{G}$. Using (1.2) we get

$$
y+\pi_{n} x_{n}^{G}=y+\left(x_{n}^{G}-\pi_{n} y\right)=x_{n}^{G}+\left(1-\pi_{n}\right) y .
$$

Thus $x_{n}^{M}$ is a modified form of both $x_{n}{ }^{S}$ and $x_{n}^{G}$. It can also be seen that $x_{n}^{M}$ satisfies the equation

$$
x_{n}^{M}-\pi_{n} \pi_{n} x_{n}^{M}=y \text {. }
$$

In the next section we show that $x_{n}^{M}$ converges to the solution $x$ of (1.1) faster that $x_{n}^{G}$ whenever $x_{n}{ }^{S}$ and $x_{n}{ }^{K}$ converge faster than $x_{n}^{G}$ - we have already mentioned that there are cases when the latter situation occurs.

\section{Main Results}

We recall the definitions from Section 1:

$$
\begin{aligned}
& x_{n}^{S}=y+T x_{n}^{G} \\
& x_{n}{ }^{K}=y+z_{n},
\end{aligned}
$$

where $z_{n}$ is the solution of $z_{n}-\pi_{n} T z_{n}=\pi_{n} T y$, and 


$$
x_{n}^{M}=x_{n}^{G}+\left(1-\pi_{n}\right) y \text {. }
$$

THEOREM 2.1. For $y \in X$, let $x$ be the solution of (1.1). Then

$$
\left\|x-x_{n}^{M}\right\| \leq\left\|\pi_{n}\right\|\left\|x-x_{n}^{S}\right\|+\left\|1-\pi_{n}\right\|\left\|x-x_{n}^{K}\right\| \text {. }
$$

Proof. The crucial point of the proof is the observation

$$
x_{n}^{K}=\pi_{n} x_{n}^{K}+\left(1-\pi_{n}\right) y \text {. }
$$

This follows from the definition of $x_{n}{ }^{K}$ :

$$
\left(1-\pi_{n}\right) x_{n}^{K}=\left(1-\pi_{n}\right)\left(y+z_{n}\right)=\left(1-\pi_{n}\right) y .
$$

Then we have

$$
\begin{aligned}
x_{n}{ }^{K}-x_{n}{ }^{M} & =\left(\pi_{n} x_{n}{ }^{K}+\left(1-\pi_{n}\right) y\right)-\left(x_{n}{ }^{G}+\left(1-\pi_{n}\right) y\right) \\
& =\pi_{n}\left(x_{n}{ }^{K}-x_{n}{ }^{S}\right),
\end{aligned}
$$

since $\pi_{n} x^{S}=x_{n}^{G}$. Thus ,

$$
\begin{aligned}
x-x_{n}{ }^{M} & =\left(x-x_{n}{ }^{K}\right)+\left(x_{n}{ }^{K}-x_{n}{ }^{M}\right) \\
& =\left(x-x_{n}{ }^{K}\right)+\pi_{n}\left(x_{n}{ }^{K}-x_{n}{ }^{S}\right) \\
& =\left(x-x_{n}{ }^{K}\right)+\pi_{n}\left(x_{n}{ }^{K}-x\right)+\pi_{n}\left(x-x_{n}{ }^{S}\right) \\
& =\pi_{n}\left(x-x_{n}{ }^{S}\right)+\left(1-\pi_{n}\right)\left(x-x_{n}{ }^{K}\right),
\end{aligned}
$$

so that

$$
\left\|x-x_{n}{ }^{M}\right\| \leq\left\|\pi_{n}\right\| \quad\left\|\mathrm{x}-\mathrm{x}_{\mathrm{n}}^{\mathrm{S}}\right\|+\left\|1-\pi_{n}\right\|\left\|x-x_{n}{ }^{K}\right\| .
$$

For the next result we introduce the following definition from Schock [6].

DEFINITION 2.1. Suppose that $x_{n}$ is an approximation to the solution $x=(1-T)^{-1} y$ of (1.1) obtained by an approximation method with the help of the projection $\pi_{n}$. The method is said to be converging if $\lim _{n \rightarrow \infty}\left(x-x_{n}\right)=0$ for all $y \in X$. A converging method is arbitrarily slowly converging if for each monotone decreasing positive null sequence $\left(w_{n}\right)$, that is $w_{n} \rightarrow 0$, there is a $y \in X$ such that $\left(\left\|x-x_{n}\right\|\right)$ 
converges more slowly than $\left(w_{n}^{\prime}\right)$, that is $\lim _{n \rightarrow \infty}\left(\left\|x-x_{n}\right\| / w_{n}\right)=\infty$. Otherwise the method is said to converge uniformly.

THEOREM 2.2. Let $X$ be a Hilbert space and let $\pi_{n}$ 's be orthogonal projections. Then we have the following:

(1) $\left\|x-x_{n}^{M}\right\| \leq 2 \max \left\{\left\|x-x_{n}^{S}\right\|,\left\|x-x_{n}^{K}\right\|\right\}$

(2) If $T$ is a compact operator, then the 'modified projection method' converges uniformly.

(3) If $T$ is a compact self-adjoint operator, then the orders of convergence of $x_{n}{ }^{S}, x_{n}^{K}$ and $x_{n}^{M}$ are the same.

Proof. The result in (1) follows directly from Theorem 2.1 by noting that for an orthogonal projection $\pi_{n},\left\|\pi_{n}\right\|=\left\|1-\pi_{n}\right\|=1$.

(2) It is proved in schock ([6], Theorem 3.1 (1)) that if $T$ is a compact operator, then the iterated Galerkin method and the Kantorovich method converge uniformly. Hence by Theorem 2.1 , the result follows.

(3) Considering the 'remainder operators' $R_{n}^{S}, R_{n}^{K}$ as $R_{n}{ }^{S} x=x-x_{n}^{S}, R_{n}^{K} x=x-x_{n}^{K}$, it is shown in Schock [6, Theorem 3.1

(2)] that, if $T$ is compact and self-adjoint, then there exists a constant $c \geq 1$ such that $\frac{1}{c}\left\|R_{n}^{S}\right\| \leq\left\|R_{n}^{K}\right\| \leq c\left\|R_{n}^{S}\right\|$. From this relation and Theorem 2.1, we get

$$
\begin{aligned}
& \left\|x-x_{n}^{M}\right\|=0\left(\left\|R_{n}^{S}\right\|\right)=\left\|x-x_{n}^{S}\right\|, \\
& \left\|x-x_{n}^{M}\right\|=o\left(\left\|R_{n}^{K}\right\|\right)=\left\|x-x_{n}^{K}\right\| .
\end{aligned}
$$

Computational remarks. Suppose that the range of the projection $\pi_{n}$ has dimension $n$. Let $e_{1}, \ldots, e_{n}$ be a basis of range $\pi_{n}$ and $e_{1}^{*}, \ldots, e_{n}^{*}$ in $X^{*}$ an adjoint basis, that is $\left\langle e_{i}, e_{j}^{*}\right\rangle=\delta_{i j}$. Then $\pi_{n}$ is given by

$$
\pi_{n} x=\sum_{i=1}^{n}\left\langle x, e_{i}^{*}\right\rangle e_{i}, \quad x \in X .
$$

In the Galerkin method we are required to find $\left(\alpha_{1}, \ldots, \alpha_{n}\right) \in \mathbb{C}^{n}$ such 
that $x_{n}^{G}=\sum_{i=1}^{n} \alpha_{i} e_{i}$ satisfies the equation (1.2). This is equivalent to solving the system

$$
\alpha-A_{n}^{\alpha}=\gamma
$$

for $\alpha=\left(\alpha_{1}, \ldots, \alpha_{n}\right)^{\prime}$, where $A_{n}=\left(\left\langle T e_{j}, e_{i}^{*}\right\rangle\right)$ and

$\gamma=\left(\left\langle y, e_{1}^{*}\right\rangle, \ldots,\left\langle y, e_{n}^{*}\right\rangle\right)^{\prime}$. (Here $\alpha$ and $\gamma$ are column vectors.)

Once the equation $(*)$ is solved, the iterated Galerkin approximation $x_{n}{ }^{S}$ is given by

$$
x_{n}^{S}=y+\sum_{i=1}^{n} \alpha_{i} T e_{i}
$$

To obtain the Kantorovich approximation $x_{n}{ }^{K}$, we have first to solve (1.4), that is, to find $\left(\beta_{1}, \ldots, \beta_{n}\right) \in \mathbb{Q}^{n}$ such that $z_{n}=\sum_{i=1}^{n} \beta_{i} e_{i}$ satisfies (1.4). This is equivalent to solving the system

$$
\beta-A_{n} \beta=\delta \text {, }
$$

for $\beta=\left(\beta_{1}, \ldots, \beta_{n}\right)^{\prime}$, where $\delta=\left(\left\langle T y, e_{1}^{*}\right\rangle, \ldots,\left\langle T y, e_{n}^{*}\right\rangle\right)$. Then

$$
x_{n}^{K}=y+\sum_{i=1}^{n} \beta_{i} e_{i}
$$

Now, knowing the solution $\alpha=\left(\alpha_{1}, \ldots, a_{n}\right)^{\prime}$ of $(*)$, the modified projection approximation $x_{n}{ }^{M}$ is given by

$$
x_{n}^{M}=y+\sum_{i=1}^{n} \alpha_{i} e_{i}-\sum_{i=1}^{n}\left\langle y, e_{i}^{*}\right\rangle e_{i}
$$

Thus, the modified projection approximation $x_{n}^{M}$ requires no more computation than $x_{n}^{G}$, except for some additions and subtractions; whereas for $x_{n}{ }^{S}$ and $x_{n}{ }^{K}$ we need to calculate $T e_{i}$ and $\left\langle T y, e_{i}^{*}\right\rangle$, respectively - these quantities of ten require further numerical approximations. For example suppose that $T$ is a compact integral operator on $c[a, b]$ (the space of continuous functions on $[a, b]$ with supremum norm), given by 


$$
(T x)(s)=\int_{a}^{b} k(s, t) x(t) d t, \quad a \leq s \leq b .
$$

If $\pi_{n}$ is an interpolatory projection associated with a mesh $a=t_{0}<t_{1}<\ldots<t_{n-1}<t_{n}=b$, then $T e_{i}$ and $\left\langle T y, e_{i}^{*}\right\rangle$ are the integrals.

$$
\int_{a}^{b} k(s, t) e_{i}(t) d t \text { and } \int_{a}^{b} k\left(t_{i}, t\right) y(t) d t
$$

respectively. If $\pi_{n}$ is an orthogonal projection with $e_{i}^{*}=e_{i}$ then $\left\langle T y, e_{i}^{*}\right\rangle$ is the double integral

$$
\int_{a}^{b} \int_{a}^{b} k(s, t) y(t) e_{i}(s) d t d s .
$$

All these above integrals are, often, computed by numerical quadrature rules, which introduce errors in the approximations $x_{n}^{S}$ and $x_{n}^{K}$-these errors, in general, depend on the smoothness of the kernel $k(s, t)$ and the given function $y(t)$. Error analysis for the approximations $x_{n}^{G}$ and $x_{n}^{S}$, when the integrals are approximated by quadrature rules, are considered by Joe in [2] and [3].

\section{References}

[1] G.A. Chandler, Superconvergence of numerical solutions to second kind integral equations. (Ph.D. Thesis, Australian National University, Canberra, 1979).

[2] S. Joe, "Discrete collocation methods for second kind Fredholm integral equations", SIAM J. Numer. Anal. 22 (1985), 1167-1177.

[3] S. Joe, "Discrete Galerkin methods for Fredholm integral equations of the second kind", IMA J. Numer. Anal. (to appear).

[4] E. Schock, "Uber die Konvergenzgeschwindigkeit projektiver Verfahren I, II ", Math. 2. 120 (1971) 148-156; 127 (1972), 191-198.

[5] E. Schock, "Galerkin-like methods for equations of the second kind", $J$. Integrat Equations 4 (1982), 361-364.

[6] E. Schock, "Arbitrarily slowly convergence, uniform convergence and superconvergence of Galerkin-like methods", IMA J. Numer. Anal. 5 (1985), 153-160. 
[7] I.H. Sloan, "Improvement by iteration for compact operator equations", Math. Comp. 30 (1976), 758-764.

[8] I.H. Sloan, E. Noussair and J. Burn, "Projection methods for equations of the second kind", J. Math. Anal. Appl. 69 (1979), 84-103.

Department of Mathematics

Indian Institute of Technology

Powai, Bombay 400 076, INDIA

Department of Mathematics,

Goa University,

Bambolim,

Santa-Cruz,

P.O., Goa - 403 005, INDIA 